\title{
The Regulation of Brassinolide Biosynthesis and Physiological Effects in Grape Berries
}

Li Jiajia, Li Xiangyi, Wang Lei $\unrhd$, Wang Shiping

Department of Plant Science, School of Agriculture and Biology, Shanghai Jiao Tong University, Shanghai, 200240, China

$\nabla$ Corresponding author email: leiwang2016@sjtu.edu.cn

Molecular Plant Breeding, 2021, Vol.12, No.33 doi: $\underline{10.5376 / \mathrm{mpb} .2021 .12 .0033}$

Received: 11 Jun., 2021

Accepted: 21 Oct., 2021

Published: 30 Oct., 2021

Copyright (C) $2021 \mathrm{Li}$ et al., This article was first published in Molecular Plant Breeding in Chinese, and here was authorized to translate and publish the paper in English under the terms of Creative Commons Attribution License, which permits unrestricted use, distribution, and reproduction in any medium, provided the original work is properly cited.

Preferred citation for this article:

Li J.J., Li X.Y., Wang L., and Wang S.P., 2021, The regulation of brassinolide biosynthesis and physiological effects in grape berries, Molecular Plant Breeding, 12(33): 1-10 (doi: $10.5376 / \mathrm{mpb} .2021 .12 .0033$ )

\begin{abstract}
Brassinolide is an important plant hormone regulating plant growth and development, promoting cell division and elongation, photosynthesis and respiration, photomorphogenesis, stress resistance improvement, transpiration reduction and make a very important contribution to the improvement of grape fruit yield and quality. In this research, key enzymes and influencing factors in brassinolide biosynthetic pathway and the effects of brassinolides on grape fruit yield and quality were reviewed, the physiological impacts of brassinolide on plant photosynthesis, respiration, transpiration, and photomorphogenesis were also summarized. Meanwhile, the interaction network between brassinolide and other plant endogenous hormones was reviewed, opinions that future research direction should be focused on the synthesis of related transcription factors and interaction mechanism between brassinolides and other plant hormones as well as research and development of new efficient brassinolides plant regulators were put forward.
\end{abstract}

Keywords Grape; Brassinolide; Synthesis; Physiological effect; Interaction mechanism

Brassinolide (BR) is a kind of trace and efficient plant hormones, which can regulate plant growth and development and is widely distributed in plants (Grove et al., 1979), so it is also known as the sixth class of hormones. At present, it is known that BR has physiological functions such as promoting cell division and growth, improving plant resistance, balancing plant vegetative growth and reproductive growth, promoting plant photosynthesis, and increasing yield (Zhao and Wang, 1986, Discovery of Nature (3): 133-136). Previous studies have shown that BR could significantly promote the growth of watermelon (Citrullus lanatus (Thunb.) Matsum. et Nakai) seedlings (Wang et al., 1994), and promote the growth of maize (Zea mays L.) roots (Kong and Zhang, 1998). Moreover, BR could significantly promote the formation of drought resistance, cold resistance, high temperature resistance, salt resistance, drug resistance and disease resistance of plants (Wang et al., 2005).

In the study of BR biosynthesis pathway, it was found that the precursor of BR synthesis was $\mathrm{C}_{30}$ derivatives of phytosterol (Cycloartenol), which generated intermediates Campesterol through methylation and isomerization. Finally, BR was generated through the early and late C6 oxidation pathways. BR also plays an important role in signal transduction. Studies have shown that BR signal regulates the expression of downstream genes under the action of a series of proteins and enzymes after being perceived by the cell membrane receptor BRI1/BAK1, and further regulates the growth and development of plants (Fujioka and Yokota, 2003).

Plant growth and development are jointly affected by a variety of hormones. Some hormones show synergism, while others show antagonism. BR can regulate ethylene production by synergism with auxin, to achieve the purpose of regulating fruit maturation (Wan, 2007). At the same time, BR and abscisic acid also show synergism, which can regulate the formation of anthocyanins in grape berries by promoting the increase of soluble solids in fruits and improve the ability of plants to stress by regulating stomatal movement ( $\mathrm{Li}$ et al., 2018). In addition, BR and salicylic acid also show synergism, which can improve the stress resistance of tomato (Lycopersicon esculentum Mill.) seedlings by regulating the activity of membrane protective enzymes (Wan, 2007). 
Grape (Vitis vinifera L.) is a fruit tree with a wide planting area in the world, which is widely used in brewing because of its sweet and sour taste and suitable crushing. In recent years, BR has been widely used in grape cultivation practice, contributing to the improvement of grape yield and quality. The results showed that BR could improve the size and yield of grape berries and clusters, improve the resistance of grape berries to stress, and increase the content of photosynthetic pigment in grape (Bajguz and Tretyn, 2003). With the improvement of analytical techniques, the application of genomics research methods, and the development of transcriptomics, BR has been widely used in grape cultivation. In this study, we summarized the molecular mechanism of BR biosynthesis and its physiological effects on grape berries quality, and provided new ideas for the future study of interaction between brassinolide and other plant hormones.

\section{Brassinolide in Grape Berries}

\subsection{Brassinolide and its classification}

American scientist Mitchell (1970) first found that plant pollen contains a large amount of BR. Subsequently, Mandava et al. (1973) purified BR by extraction and elution, and speculated the stereochemical structure of BR (Figure 1). BR is widely present in plants and is the only plant hormone similar to animal hormones. It is homologous to the steroid $5 \alpha$-reductase gene in mammals, which means that the possible metabolic synthesis and signal transduction pathways of BR can be inferred by analyzing the mechanism of animal hormones in the future.

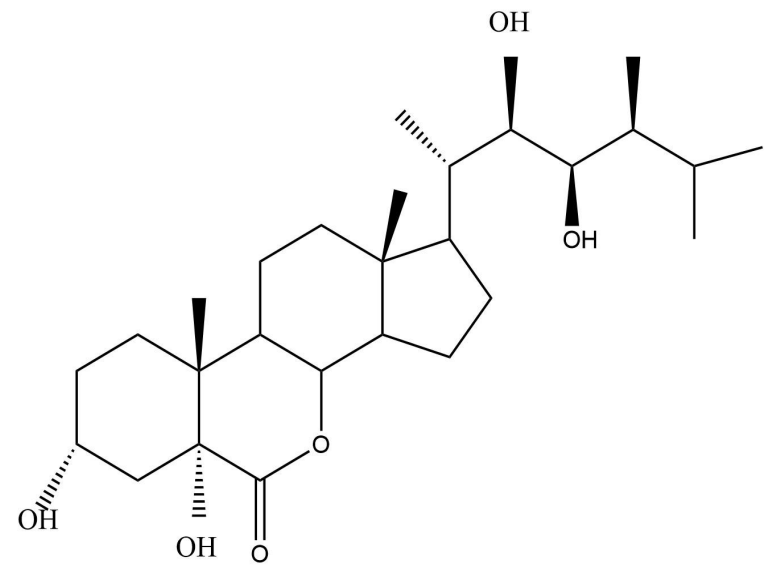

Figure 1 Brassinolide (Grove et al., 1979)

BR is a steroid compound. According to the number of carbon atoms in steroid molecules, all BRs can be divided into three categories: $\mathrm{C}_{27}, \mathrm{C}_{28}$ and $\mathrm{C}_{29}$ (Fujioka and Yokota, 2003). With the improvement of hormone analysis technology, more than 70 kinds of brassinosteroids have been found. In Typhaceae Juss., the main forms of BR are Typhasterol (Figure 2A) and Teasterone (Figure 2B) (Bajguz and Tretyn, 2003). In rice (Oryza sativa L.) of Poaceae, the main forms of BR are Brassinolide (Figure 2C), Castasterone (Figure 2D) and Dolichosterone (Figure 2E). In Arabidopsis thaliana of Brassicaceae, the main forms of BR are Brassinolide (Figure 1) and 6-Deoxocastasterone (Figure 2F). In Citrus reticulata Blanco L. of Rutaceae, the main forms of BR are Brassinolide (Figure 2C), Castasterone (Figure 2D), Typhasterol (Figure 2A) and Teasterone (Figure 2B).

\subsection{Biosynthesis pathway of brassinolide the early and late C-6 oxidation pathways}

The research on the biosynthesis pathway of BR is mainly focused on the mutants. And it has been found that the biosynthesis pathway of BR can be divided into the early and late C6 oxidation pathways. Suzuki et al. (1995) found that Campesterol was the starting substance of BR synthesis, and 6-Oxocampestanol was finally obtained by oxygenation, hydroxylation and oxidation. Fujioka (1997) found that the product will continue to undergo hydroxylation, dehydroxylation and re-hydroxylation to form BR, which is collectively referred to as the early $\mathrm{C} 6$ oxidation pathway. Furthermore, it was found that 6-Dehydrocastasterone could be directly converted into Castasterone in the cultured cells of Catharanthus roseus, which means that there is another pathway for the synthesis of brassinolide in plants, that is the late $\mathrm{C} 6$ oxidation pathway. 


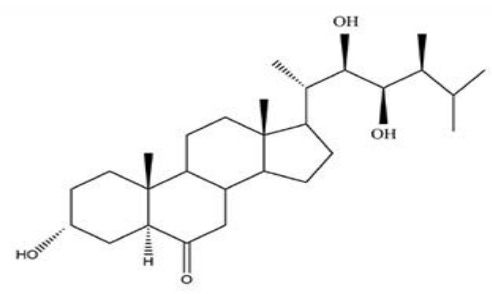

A

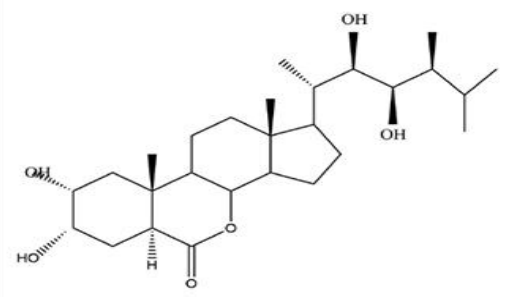

$\mathrm{C}$

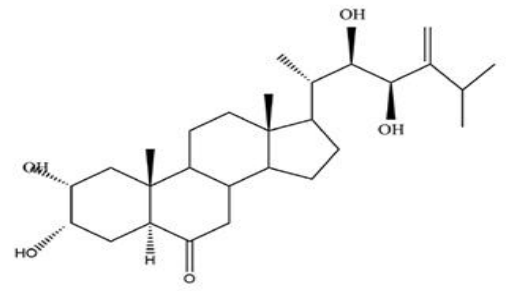

$\mathrm{E}$<smiles>CC(C)C(C)[C@H](C)[C@H](C)CC1CCC2C3CC(=O)[C@]4(C)CC(C)(C)CC[C@]4(C)C3CCC12C</smiles>

B

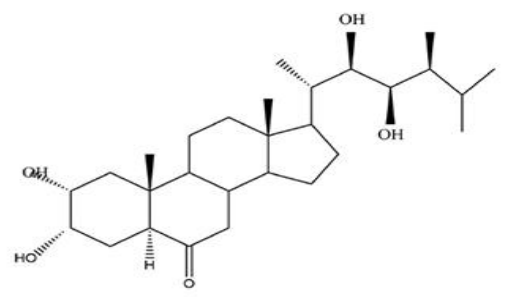

$\mathrm{D}$

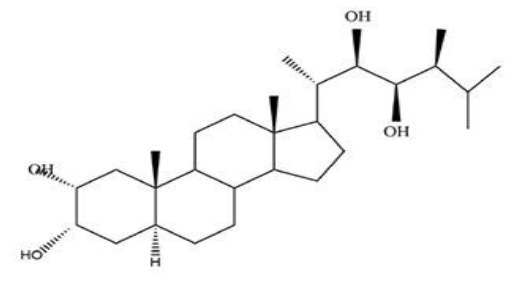

$\mathrm{F}$

Figure 2 Brassinoid steroids (Noguchi et al., 2000)

Note: A: Typhasterol; B: Teasterone; C: Brassinolide; D: Castasterone; E: Dolichosterone; F: Deoxocastasterone

The specific process of the early C6 oxidation pathway (Figure 3) is as follows: Campesterol is used as the synthesis precursor to generate Campestanol by dehydrogenation reaction, and then $6 \alpha$-Hydroxycampestanoil is generated by hydroxylation, and then 6-Oxocampestanol is generated by oxygenation reaction. After that, the two-step hydroxylation is used to generate Cathasterone and Teasterone. Then, the dehydrogenation reaction is used to generate 3-Dehydroteasterone, and the further reaction is used to generate Typhasterol. Finally, the hydroxylation is used to generate Castasterone, and the further oxidation is used to generate Brassinolide (Noguchi et al., 2000).

The specific process of the late C6 oxidation pathway (Figure 3) is as follows: 6-Dehydroteasterone is used as the synthesis precursor to generate 3-Dehydro-6-Deoxoteasterone by dehydrogenation reaction, and then 6-Oeoxocastasterone is synthesized by hydroxylation, further dehydrogenation is used to generate Castasterone, and finally dehydrogenation to generate Brassinolide (Noguchi et al., 2000).

\section{Biosynthesis Regulation of Brassinolide}

\subsection{Synthesis and optical signal regulation of brassinolide}

Plant photomorphogenesis is a low-energy response, and most BR-deficient mutants show leaf curl and yellow (Hong et al., 2002). Luo et al. (2010) isolated and identified the transcription factor $\mathrm{GATA}_{2}$ in Arabidopsis thaliana, which regulates plant photomorphogenesis. Its role is to directly participate in the process of BR regulating light signal transduction, and then participate in the regulation of plant photomorphogenesis. 


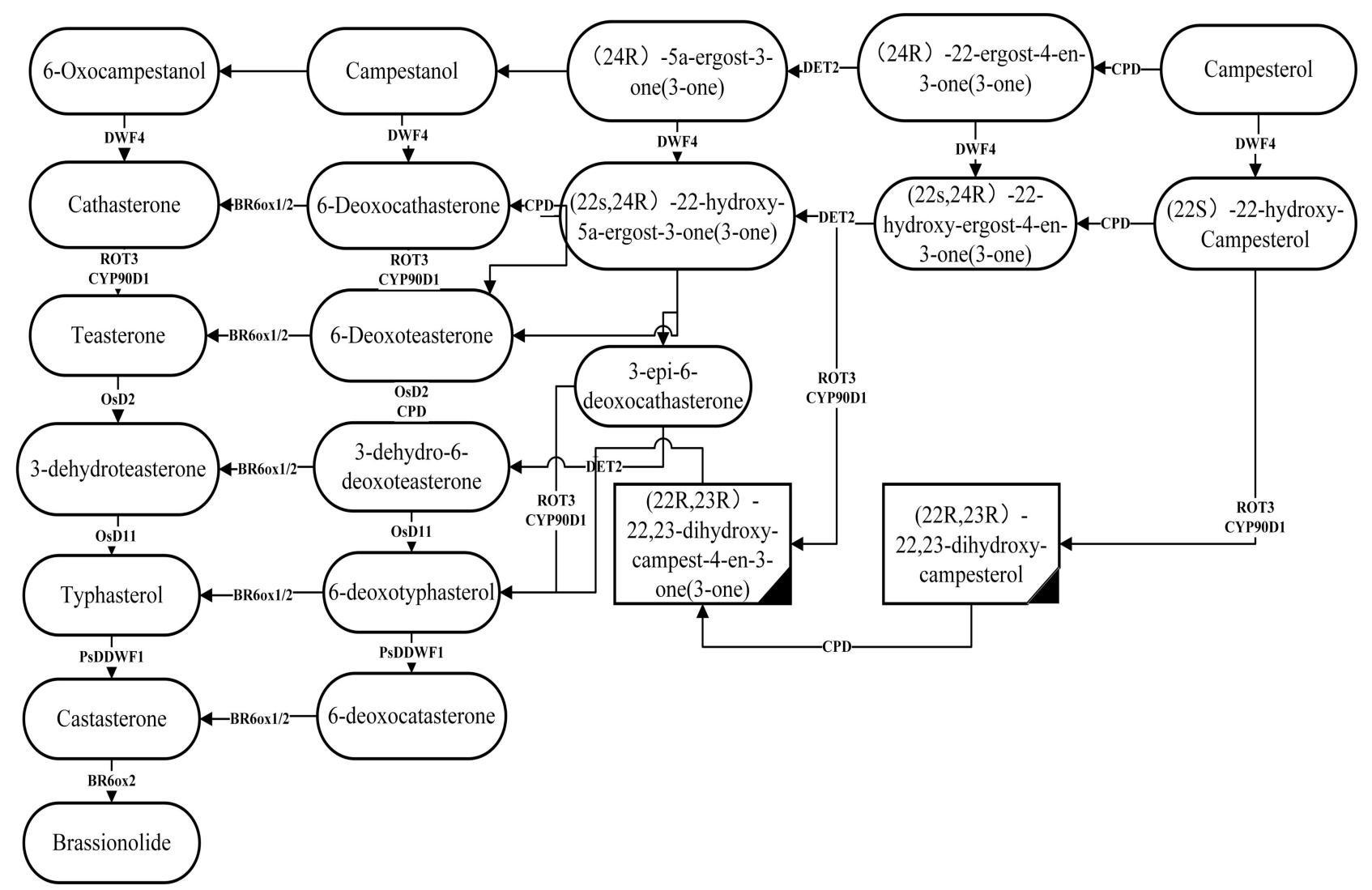

Figure 3 Anabolic pathway of brassinolide (Noguchi et al., 2000)

\subsection{Regulatory genes for brassinolide biosynthesis}

In the process of BR signal transduction, receptor kinase BRI1 and BAK1 play a decisive role in the signal pathway. Overexpression of BRI1 will increase the sensitivity of plants to BR, and the binding with BR is stronger. The mechanism of BAK1 and BRI1 action can be summarized as follows: BR binding protein binds to the extracellular domain of BRI1, thereby mediating BR signal transduction. Mutants of BRI1 homologs use a new activation method to initiate BR signal transduction after binding to BR. Li et al. (2002) found that BAK1 and BRI1 have the same expression pattern, and both proteins are located on the plasma membrane, so they can interact. In addition, BAK1 and BRI1 can be phosphorylated to each other, and BAK1 shows a higher phosphorylation level in the presence of BRI1. Tang et al. (2008) studied BRI1 signal transduction mediated by BSK genes in Arabidopsis thaliana and found that BAK1 is most likely to mediate the activation of BRI1 kinase rather than the signal transduction of specific downstream components of the BR signal pathway. On the contrary, $B S K$ directly mediates the signal transduction of the response from BRI1 to downstream BR. In the study of BR signal transduction pathway, it was found that BAK1 regulated the transport of BRI1, and the overexpression of BRI1 and BAK1 co-receptors in protoplasts increased the internal accumulation of BRI1. However, the inherent limitations of the protoplast system limited the attempt to link functional endocytosis with BRI1 signal transduction, so this hypothesis has not been confirmed (Russinova et al., 2004).

In the process of BR signal transduction, BZR1 plays an important role in mediating the feedback inhibition of BR biosynthesis. By identifying the nuclear components of BZR1 mutant, it was found that BZR1 is a positive regulator of BR signaling pathway, mediating the feedback regulation of downstream BR physiological effects and BR biosynthesis (Wang et al., 2002). As a BR synthesis transcription inhibitor, BZR1 plays a dual role in the formation and growth of BR homeostasis. Hong et al. (2005) studied the mutant bzrl-1D of BR in Arabidopsis thaliana and found that the dominant bzrl-1D mutant could increase the accumulation of BZR1 protein, inhibit BR-insensitive bril and bin 2 mutants, and lead to insensitivity to brassinazole, an inhibitor of BR biosynthesis. These findings indicate that BZR1 plays an active role in BR signal transduction. 


\section{Physiological Effects of Brassinolide in Plants}

\subsection{Effects of brassinolide on plant growth and development}

As an important hormone in plants, BR plays an extremely important role in regulating plant growth and development, which is reflected in promoting cell elongation, cell division, plant reproductive growth and plant tropic movement.

BR increases plant height mainly by modifying cell wall and regulating gene expression of cell wall synthesis, thereby affecting cell wall formation. Cell elongation is mainly related to the synthesis of xyloglucan endotransglycosylase and expansin. It has been found that BR can promote the addition of new xyloglucan to the cell wall, lead to cell relaxation and cell elongation (Jin et al., 2014). With the help of BR mutant in Arabidopsis thaliana, Xie et al. (2011) proved that BR regulated cellulose synthase by binding to the upstream components of cellulase synthase, which also explained why BR promoted cell elongation. It was found that $\mathrm{MD}_{40}$, as a regulatory protein, has a positive regulatory effect on hypocotyl elongation in Arabidopsis thaliana, and the receptor kinase FERONIA related to pollen tube elongation is also involved in the process of BR signal transduction (Deslauriers and Larsen, 2010). Through the analysis of the leaf angle of BR-deficient mutant rice, it was found that the inclination at the leaf junction was significantly reduced, suggesting that BR could increase the leaf bending degree (Nakaya et al., 2002).

BR promoted cell division mainly in promoting leaf expansion, promoting the process of root cell cycle, regulating the number of vascular bundles and inhibiting stomatal formation. By cutting the epicotyl of soybean (Glycine $\max$ (Linn.) Merr.) and BR treatment, it has been confirmed that BR can upregulate the expression of cyclin $\mathrm{CycD} 3$ ( $\mathrm{Hu}$ et al., 2000), thus affecting cell division, and exogenous BR can expand the leaves of BR-deficient mutant plants (Nakaya et al., 2002). The effect of BR on the root system is to regulate the division of the cell quiescent center and the differentiation process of the distal stem cells. Kuppusamy et al. (2009) found that BR could also affect root hair formation and promote lateral root formation. Auxin can promote the development of xylem, so some researchers speculated that BR can also regulate the life activity of xylem. It has been confirmed that BR signal transduction receptors BRL1 and BRL3 can regulate the development of vascular bundles by regulating the differentiation ratio of phloem and xylem (Wang et al., 2001). When exogenous BR was applied to Arabidopsis thaliana, it was also found that the proportion of stomatal closure increased and stomatal development slowed, suggesting that BR regulates stomatal opening, closing and development through phosphorylation of transcription factor $S P C H$ related to stomatal development (Gudesblat et al., 2012).

BR regulates plant reproductive growth by promoting pollen tube elongation, affecting flowering time, inducing flowering, increasing yield and fruit quality. Li et al. (2011) found that the application of exogenous BR could advance the flowering time of Arabidopsis thaliana and could induce flowering of plants without vernalization and independent pathway

The directional movement of plants can be divided into gravitropism, phototropism, thigmotropism, chemotropism. At present, it has been proved that BR and auxin are antagonistic in the regulation of plant gravitropism. Exogenous BR can inhibit the gravitropism of hypocotyls by reducing the content of auxin in seedlings (Gupta et al., 2012). Further studies have found that brassinolide can induce the synthesis of ACC synthase, thereby regulating ethylene on plant epinasty growth, but whether BR has an impact on plant phototropism, chemotropism and thigmotropism is unclear.

\subsection{Effects of brassinolide on plant life activities}

The growth and development of plants are constantly carrying out various life activities, including photosynthesis, respiration, transpiration, and photomorphogenesis.

Photosynthesis is the most important metabolism activity of plant, and it has been confirmed that BR could promote the photosynthesis. The net photosynthetic rate of Cucumis melo was significantly increased by applying BR (Zhang et al., 2012). It is speculated that BR could improve the capture of light energy by leaves, thus promoting the process of PSII activity and carbon assimilation and improving fruit yield and quality. 
Respiration is also an indispensable activity for plant growth and development. It has been confirmed that BR could improve plant respiration by regulating the activities of various enzymes in TCA cycle. The application of exogenous BR in cucumber seedling roots showed that the expression of aerobic respiration isoenzyme was significantly increased, which greatly solved the problem of hypoxia during seedling growth and development $(\mathrm{Lu}$ et al., 2012).

Transpiration is the main way of plant heat dissipation. The application of BR to Hippophae rhamnoides seedlings under drought stress showed that the damage caused by stress was reduced, the transpiration rate was reduced, and the drought resistance was enhanced. It is further speculated that BR may promote stomatal closure and slow down the rate of water metabolism (Han and Li, 2012).

\subsection{Effect of brassinolide on plant quality}

Quality is an important index to measure plant growth and development, which includes morphological indexes and physiological indexes. Morphological indexes include dry and fresh weight of aboveground part, dry and fresh weight of underground part, plant height, stem diameter and leaf area. Physiological indexes include soluble sugar, protein, photosynthetic pigment content, polyphenol content and anthocyanin content. Since BR has synergism with auxin and ethylene, it is speculated that BR also plays an important role in improving plant quality. The application of exogenous BR showed that the seeds number and grain weight of Arabidopsis thaliana seeds increased significantly, which may be related to the fact that BR could increase the activity of sucrose phosphate synthase and thus accumulate sucrose content in the plant (Huang et al., 2013). After spraying a certain amount of BR on the young leaves of Fragaria $\times$ ananassa Duch., it was found that the soluble sugar and soluble protein of the fruit were significantly increased, and the maturity period was advanced, and the fruit quality was significantly improved (Aaby et al., 2012).

The application of epbrassinolide (EBR) once in young leaf stage of muskmelon increased stomatal conductance and photosynthetic indexes compared with the application of 2 and 3 times. The photosynthetic indexes of EBR twice and 3 times in fruit stage of muskmelon were significantly higher than those of spraying once (Shi et al., 2015). This is because BR could slow down the decrease of photosynthetic pigment activity of mesophyll cells by regulating stomatal conductance, thereby maintaining photosynthetic rate to a certain extent. The results showed that after spraying BR on peach (Amygdalus persica L.) at mature stage, the content of soluble solid, soluble sugar, sucrose and fructose increased. It was speculated that BR could regulate the vegetative growth of fruit trees and effectively promote the accumulation of nutrients (Lin et al., 2016). After applying BR to grape 'Hongdiqiu' at young fruit stage and expansion stage, it was found that total phenol content in peel increased significantly, sugar acid ratio, Vc content and flavonoid content increased, which confirmed that BR could promote the distribution and accumulation of photosynthetic products in plants (Feng, 2014). After spraying BR on tomato at mature stage, it was found that the soluble sugar content and sugar-acid ratio increased, but the lycopene had no significant difference. It was speculated that BR could maintain the balance of sucrose synthesis and decomposition by regulating the activity of sucrose synthase, so that sucrose was more likely to be transported to the fruit, and the fruit quality was improved accordingly (Li et al., 2014). Ma et al. (2012) found that low concentration of BR could promote the accumulation of anthocyanin in grape skin after the application of EBR at full flowering stage. It was speculated that exogenous BR could regulate the synthesis of anthocyanin by interacting with other plant hormones. At the same time, it was found that the expression of $P A L$ and $U F G T$, the key genes of anthocyanin synthesis pathway, was significantly increased, which further proved that BR regulated the production of anthocyanin. These studies further demonstrated that BR plays an important role in improving fruit quality.

\subsection{Effect of brassinolide on plant stress resistance}

Under the adverse environmental conditions such as drought, freezing injury, high temperature and high salt, plants will show some phenomena, such as slower growth, weaker photosynthesis, weaker respiration, lower quality, lower yield and so on (Zhang, 2000). In general, the ability of plants to resist adversity is called stress resistance. The stress resistance of plants is mainly related to the activity of radical scavenger, and the physiological effect of BR is similar to that of abscisic acid. It has been confirmed that BR plays an important role 
in improving the stress resistance of plants. Its mechanism is to increase the activity of peroxidase, catalase and glutathione reductase, so as to improve the speed of radical scavenger and protect plants from stress. At the same time, BR can also increase the content of proline, an osmotic adjustment substance in plants, so as to reduce the degree of stress in plants (Zhu, 1992). In recent years, studies on the interaction between BR and other plant hormones to improve plant stress resistance have been more and more extensive, and it has been confirmed that abscisic acid and BR can synergistically improve plant stress resistance. BR could upregulate the expression of abscisic acid synthesis genes to improve the plant drought and low temperature resistance (Kagale et al., 2007). After applying BR to the Malus pumila under drought stress, it was found that the leaf growth accelerated, the fruit setting rate increased, and the fruit yield increased significantly (Li et al., 2003), which further indicated that the application of BR could alleviate the degree of drought stress.

Freezing resistance refers to the ability of plants to resist freezing injury. The damage of plants under low temperature environment is divided into cold injury and freezing injury. Cold injury will lead to slow growth and development of plants, decrease of photosynthesis rate and quality. Freezing injury will lead to the destruction of cell membrane, the destruction of osmotic balance in vivo and the death of plants. Cell damage caused by freezing injury is due to membrane peroxidation, resulting in more malondialdehyde, which causes damage to cell membrane. Further study found that the relative conductivity of transgenic mustard (Brassica juncea (L.) Czern. et Coss.) decreased, the proline accumulation increased, and the expression of $D W F_{4}$ gene significantly increased after BR application in cold state (Lan, 2016), indicating that the key gene $D W F_{4}$ in brassinolide synthesis pathway played an important role in enhancing plant stress resistance. These studies have confirmed that BR can increase plant tolerance to cold stress by promoting the synthesis of osmotic regulators and increasing the activity of membrane protective enzymes.

Salt tolerance refers to the ability of plants to resist salt stress. Usually, plant growth and development will be affected under high salt stress, resulting in cell membrane damage, osmotic imbalance, and yield decline. The salt tolerance of wheat seedlings was significantly improved after the application of exogenous EBR. The researchers speculated that BR up-regulated the expression of WRKY transcription factor related to salt tolerance and regulated salt tolerance of plants (Li et al., 2020). Kou (2016) found that the antioxidant enzyme content of Medicago sativa L. was significantly improved after the application of exogenous EBR, and the ability of plants to tolerate salt stress was enhanced. It was speculated that BR can improve the adaptability of plants to salt stress by regulating the activity of cell membrane protective enzymes.

\section{Application of Brassinolide in Grape and Its Future Research Direction}

Grape as an important fruit tree, how to improve its fruit quality has been a research hotspot. BR has been widely used in grape production because it can promote plant growth, improve photosynthetic capacity and drought resistance. After the application of BR to grape berries, it was found that the fruit maturity was advanced, while the fruit maturity was delayed after the application of BR inhibitors, which directly proved that BR was involved in the regulation of fruit maturity (Symons et al., 2006). Vergara et al. (2018) found that the color of fresh grapes became bright, and the fruit quality was improved after applying BR analogues to grapes. Zheng et al. (2020) found that the fructose content increased, the organic acid content decreased, and the anthocyanin content increased significantly after BR treatment. Further analysis showed that BR could inhibit the activity of $H M G R$ and regulate the color of fruit with $V \nu H M G R$, thus promoting the formation of aroma and other quality characteristics of fruit. Other research results showed that the yield, soluble sugar content and antioxidant capacity of grapes were significantly increased after BR application (Babalık et al., 2019).

Plant growth regulator is trace and high efficiency and plays an extremely important role in agricultural production. At present, naphthalene acetic acid, paclobutrazol, indole-3-acetic acid, ethrel and chlorocholine chloride are widely used in agricultural production. However, these plant growth regulators cannot promote plant growth and development for a long time at present, and the form of action is single, so it is difficult to comprehensively regulate plant growth and reproduction. As a new plant hormone that can interact with a variety of plant hormones, BR has a wide range of physiological effects and could comprehensively promote plant growth 
and metabolic activities. Therefore, future studies should focus on the interaction mechanism between BR and other hormones to develop BR-type growth regulators. BR could interact with auxin, gibberellin, abscisic acid and ethylene (Tanaka et al., 2003; Ma et al., 2012), but it is not clear whether BR could interact with cytokinin, salicylic acid and jasmonic acid. And the regulatory network of BR and other plant hormones will also be the direction of future research. It was found that the problem of pesticide residues should be solved (Zhang, 2011) by analyzing the application status of BR in recent years. The advantage of BR is that it has the characteristics of less dosage and quick effect, which can reduce pesticide residues well. Therefore, the development of new BR growth regulators is a new direction of plant hormone research in the future and has a broad prospect.

\section{Authors' contributions}

WL conceived and directed of the project. LJJ collected the materials and drafted manuscript. LXY and WSP reviewed the manuscript. All authors read and approved the final manuscript.

\section{Acknowledgments}

This study was supported by Shanghai Science and Technology Development Project (HNK(2019)No.1-5).

\section{Reference}

Aaby K., Mazur S., Nes A., and Skrede G., 2012, Phenolic compounds in strawberry (Fragaria x ananassa Duch.) fruits: Composition in 27 cultivars and changes during ripening, Food Chemistry, 132(1): 86-97

https://doi.org/10.1016/j.foodchem.2011.10.037

PMid:26434267

Babalık Z., Demirci T., Aşcı Ö.A., and Baydar N.G., 2019, Brassinosteroids modify yield, quality, and antioxidant components in Grapes (Vitis vinifera cv. Alphonse lavallée), Journal of Plant Growth Regulation, 1-10 https://doi.org/10.1007/s00344-019-09970-5

Bajguz A., and Tretyn A., 2003, The chemical characteristic and distribution of brassinosteroids in plants, Phytochemistry, 62(7): 1027-1046 https://doi.org/10.1016/S0031-9422(02)00656-8

Deslauriers S.D., and Larsen P.B., 2010, FERONIA is a key modulator of brassinosteroid and ethylene responsiveness in Arabidopsis hypocotyls, Mol. Plant., 3: $626-640$

https://doi.org/10.1093/mp/ssq015

PMid:20400488

Feng X.X., 2014, Effects of brassinolide on physiological and biochemical characteristics and quality of red earth Grape, Thesis for M.S., Gansu Agricultural University, Supervisor: Yang J.S., pp.46-49

Fujioka S., and Yokota T., 2003, Biosynthesis and metabolism of brassinosteroids, Annual Review of Plant Biology, 54: 137-164

https://doi.org/10.1146/annurev.arplant.54.031902.134921

PMid:14502988

Fujioka S., Li J., Choi Y.H., Seto H., Takatsuto S., Noguchi T., Watanabe T., Kuriyama H., Yokata T., Chory J., and Sakurai A., 1997, The Arabidopsis de-etiolated 2 mutant is blocked early in brassinosteroid biosynthesis, Plant Cell, 9: 1951- 1962

https://doi.org/10.1105/tpc.9.11.1951

PMid:9401120 PMCid:PMC157049

Grove M.D., Spencer G.F., Rohwedder W.K., Mandava N., Worley J.F., Warthen J.D., and Cook J.C., 1979, Brassinolide, a plant growth-promoting steroid isolated from Brassica napus pollen, Nature, 281(5728): 216-217

https://doi.org/10.1038/281216a0

Gudesblat G.E., Schneider-PizońJ., Betti C., Mayerhofer J., Vanhoutte I., Van Dongen W., and Russinova E., 2012, SPEECHLESS integrates brassinosteroid and stomata signalling pathways, Nature cell biology, 14(5): 548-554

https://doi.org/10.1038/ncb2471

PMid:22466366

Gupta A., Singh M., Jones A.M., and Laxmi A., 2012, Hypocotyl directional growth in Arabidopsis: a complex trait, Plant physiology, 159(4): 1463-1476 https://doi.org/10.1104/pp.112.195776 PMid:22689891 PMCid:PMC3425191

Han G., and Li K., 2011, Effects of brassinolide on photosynthesis of pygmy apricot under drought stress, Xibei Linxueyuan Xuebao (Journal of Northwest Forestry University), 26(4):27-32

Hong Z., Ueguchi-Tanaka M., Shimizu-Sato S., Inukai Y., Fujioka S., Shimada Y., and Matsuoka M., 2002, Loss-of-function of a rice brassinosteroid biosynthetic enzyme, C-6 oxidase, prevents the organized arrangement and polar elongation of cells in the leaves and stem, The Plant Journal., 32(4): 495-508

https://doi.org/10.1046/j.1365-313X.2002.01438.x

PMid: 12445121 
Hu Y.X., Bao F., and Li J.Y., 2000, Promotive effect of brassinosteroids on cell division involves a distinct CycD3-induction pathway in Arabidopsis, The Plant Journal, 24(5): 693-701

https://doi.org/10.1046/j.1365-313x.2000.00915.x

PMid: 11123807

Huang H.Y., Jiang W.B., Hu Y.W., Wu P., Zhu J.Y., Liang W.Q., and Lin, W.H., 2013, BR signal influences Arabidopsis ovule and seed number through regulating related genes expression by BZR1, Molecular plant, 6(2): 456-469

https://doi.org/10.1093/mp/sss070

PMid:22914576

Jin H., Do J., Shin S.J., Choi J.W., Im Choi Y., Kim W., and Kwon M., 2014, Exogenously applied 24-epi brassinolide reduces lignification and alters cell wall carbohydrate biosynthesis in the secondary xylem of Liriodendron tulipifera, Phytochemistry, 101: 40-51

https://doi.org/10.1016/j.phytochem.2014.02.003 PMid:24582278

Kagale S., Divi U.K., Krochko J.E., Keller W.A., and Krishna P., 2007, Brassinosteroid confers tolerance in Arabidopsis thaliana and Brassica napus to a range of abiotic stresses, Planta, 225(2): 353-364

https://doi.org/10.1007/s00425-006-0361-6

PMid:16906434

Kong X.S., and Zhang M.X., 1998, Effects of brassinolide and plobulozole on seed germination and seedling growth of maize (Zea mays L.), Zhongguo Nongxue Tongbao (Chinese Agricultural Science Bulletin), (2): 3-5

Kou J.T., 2016, 2, 4-epbrassinolide induced physiological response of alfalfa to salt tolerance, Dissertation for Ph.D., Gansu Agricultural University, Supervisor: Shi S.L., pp.94

Kuppusamy K.T., Chen A.Y., and Nemhauser J.L., 2009, Steroids are required for epidermal cell fate establishment in Arabidopsis roots, Proc. Natl. Acad. Sci. USA., 106: 8073-8076

https://doi.org/10.1073/pnas.0811633106

PMid:19416891 PMCid:PMC2683123

Lan C.Y., 2016, Effects of over-expression of ATDWF4 gene on growth, development and cold tolerance of Brassica juncea, Thesis for M.S., Southwest University, Supervisor: Song H.Y., pp.49-50

Li J., Wen J., and Lease K.A., 2002, BAK1, an Arabidopsis LRR receptor-like protein kinase, interacts with BRI1 and modulates brassinosteroid signaling, Cell., 110(2): 213-222

https://doi.org/10.1016/S0092-8674(02)00812-7

Li J.H., 2011, Effects of endogenous brassinolide on flowering time in Arabidopsis thaliana and its mechanism, Dissertation for Ph.D., Lanzhou University, Supervisor: An L.Z., pp.48

Li K.R., Wu F.Q., and Wang J., 2003, Effects of natural brassinolide on the growth and yield of apple in the Loess Hilly Region, Shuitu Baochi Xuebao (Journal of Soil and Water Conservation), (3): 174-177

Li N., Su X.Q., Sun J., Shu S., and Guo S.R., 2014, Effects of exogenous 24-epibrassinolide on endogenous hormone and carbohydrate metabolism in leaves and fruit of tomato under low light stress, Nanjing Nongye Daxue Xuebao (Journal of Nanjing Agricultural University), $37(3)$ : 51-56

Li Q.F., Lu J., Yu J.W., Zhang C.Q., and Liu Q.Q., 2018, Molecular mechanism of brassinolide and abscisic acid interaction in regulating plant growth and stress tolerance, Zhiwu Shengli Xuebao (Plant Physiology Communications), 54(3): 370-378

Li, Q.L., 2020, Effects of brassinolides on salt tolerance in Cucumis sativus L., Thesis for M.S., Northwest Normal University, Supervisor: Zhang, T.G., pp.49

Lin Z., Su S.C., Ma C., Bai Q., and Yang S.Y., 2016, Effects of brassinolide on photosynthesis and fruit quality of peach in Feicheng, Jingjilin Yanjiu (Nonwood Forest Research), 34(2): 73-78

Lu X.M., Sun J., Guo S.R., He L.Z., Wang L.P., and Yang Y.J., 2012, Effects of brassinolide on the expression of isozyme of aerobic respiration in roots of cucumber seedlings under hypoxic stress, Shengtaixue Zazhi (Chinese Journal of Ecology), 31(12):3070-3074

Luo X.M., Lin W.H., and Zhu S.W., 2010, Integration of light and brassinosteroid signaling pathways by a GATA transcription factor in Arabidopsis, Dev. Cell., 19(6): $872-883$ https://doi.org/10.1016/j.devcel.2010.10.023

PMid:21145502 PMCid:PMC3022420

Ma L.N., Hui Z.M., Huo S.S., Luan L.Y., Gao X., and Zhao X.L., 2012, Effects of brassinolide and abscisic acid on anthocyanin synthesis in grape fruits, Guoshu Xuebao (Journal of Fruit Science), 29(5): 830-836

Mandava N., Sidwell B. A., Mitchell J. W., and Worley J. F., 1973, Production of brassins from rape pollen, a convenient preparatory method, Industrial \& Engineering Chemistry Product Research and Development, 12(2): 138-139

https://doi.org/10.1021/i360046a009

Mitchell J.W., Mandava N., and Worley J.F., 1970, Brassins - a new family of plant hormones from rape pollen, Nature, 225, 1065-1066 https://doi.org/10.1038/2251065a 0 PMid: 16056912

Nakaya M., Tsukaya H., Murakami N., and Kato M., 2002, Brassinosteroids control the proliferation of leaf cells of Arabidopsis thaliana, Plant Cell Physiol., 43: 239-244

https://doi.org/10.1093/pcp/pcf024

PMid:11867704 
Noguchi T., Fujioka S., Choe S., Takatsuto S., Tax F.E., Yoshida S., and Feldmann K.A., 2000, Biosynthetic pathways of brassinolide in Arabidopsis, Plant Physiology., 124(1): 201-210

https://doi.org/10.1104/pp.124.1.201

PMid:10982435 PMCid:PMC59135

Russinova E., Borst J.W., Kwaaitaal M., Cano-Delgado A., Yin Y., Chory J., and de Vries S.C., 2004, Heterodimerization and endocytosis of Arabidopsis brassinosteroid receptors BRI1 and AtSERK3 (BAK1), Plant Cell, 16: 3216-3229

https://doi.org/10.1105/tpc.104.025387

PMid:15548744 PMCid:PMC535869

Shi Y., Wang Q.M., Ye H.X., Wang C., and Wang B.L., 2015, Effects of 2, 4-epbrassinolide (EBR) on photosynthetic characteristics and fruit quality of muskmelon, Henong Xuebao (Journal of Nuclear Agricultural Sciences), 29(5): 1009-1017

Suzuki H., Fujioka S., Takatsuto S., Yokota T., Murofushi N., and Sakurai A., 1995, Biosynthesis of brassinosteroids in seedlings of Catharanthus roseus, Nicotiana tabacum, and Oryza sativa. Bioscience, biotechnology, and biochemistry, 59(2): 168-172

https://doi.org/10.1271/bbb.59.168

Symons G.M., Davies C., Shavrukov Y., Dry I.B., Reid J.B., and Thomas M.R., 2006, Grapes on steroids, brassinosteroids are involved in grape berry ripening, Plant physiology, 140(1): 150-158

https://doi.org/10.1104/pp.105.070706

PMid:16361521 PMCid:PMC1326039

Tanaka K., Nakamura Y., Asami T., Yoshida S., Matsuo T., and Okamoto S., 2003, Physiological roles of brassinosteroids in early growth of Arabidopsis: brassinosteroids have a synergistic relationship with gibberellin as well as auxin in light-grown hypocotyl elongation, Journal of Plant Growth Regulation, 22(3): 259-271

https://doi.org/10.1007/s00344-003-0119-3

Tang W., Kim T.W., Oses-Prieto J.A., Sun Y., Deng Z., Zhu S., and Wang Z.Y., 2008, BSKs mediate signal transduction from the receptor kinase BRI1 in Arabidopsis, Science., 321(5888): 557-560

https://doi.org/10.1126/science. 1156973 PMid:18653891 PMCid:PMC2730546

Vergara A.E., Díaz K., Carvajal R., Espinoza L., Alcalde J.A., and Pérez-Donoso A.G., 2018, Exogenous applications of brassinosteroids improve color of red table grape (Vitis vinifera L. Cv. "Redglobe") berries, Frontiers in plant science, 9: 363

https://doi.org/10.3389/fpls.2018.00363 PMid:29681907 PMCid:PMC5897653

Wan Z.L., 2007, Effects of salicylic acid and epbrassinolide on the resistance of tomato seedlings to high temperature, Thesis for M.S., Southwest University, Supervisor: Luo Q.X., pp.38

Wang H.H., Li K.R., and Hou H.W., 2005, Research progress of brassinolide to improve plant stress resistance, Ganhan Diqu Nongye Yanjiu (Agricultural Research in the Arid Areas), (3): 213-219

Wang Y.Q., Luo W.H., Xu R.J., and Zhao Y.J., 1994, Effects of epbrassinolide on the growth and yield traits of watermelon (Watermelon), Zhiwu Shenglixue Tongxun (Plant Physiology Communications), (6): 423-425

Wang Z.Y., Nakano T., Gendron J., He J., Chen M., Vafeados D., and Chory J., 2002, Nuclear-localized BZR1 mediates brassinosteroid-induced growth and feedback suppression of brassinosteroid biosynthesis, Developmental cell, 2(4): 505-513 https://doi.org/10.1016/S1534-5807(02)00153-3

Wang Z.Y., Seto H., Fujioka S., Yoshida S., and Chory J., 2001, BRI1 is a critical component of a plasma-membrane receptor for plant steroids, Nature, 410(6826): $380-383$

https://doi.org/10.1038/35066597

PMid: 11268216

Xie L.Q., Yang C.J., and Wang X.L., 2011, Brassinosteroids can regulate cellulose biosynthesis by controlling the expression of CESA genes in Arabidopsis, Journal of experimental botany, 62(13): 4495-4506

https://doi.org/10.1093/jxb/err164

PMid:21617247 PMCid:PMC317055

Zhang L., 2011, Physiological effects of brassinolide and its development prospect, Beifang Yuanyi (Northern Horticulture), (20): 188-191

Zhang Y.P., Chen Y.Y., and Yang S.J., 2012, Effects of 2, 4-epbrassinolide on physiological and photosynthetic characteristics of muskmelon seedlings under high temperature stress, Zhiwu Shenglixue Xuebao (Plant Physiology Journal), 48(7): 683-688

Zhang, Z.B., 2000, Study on the global resistance of plants to environmental stress, Xibei Nongye Xuebao (Acta Agriculturae Boreali-occidentalis Sinica), 9(3): $112-116$

Zheng T., Dong T., Haider M.S., Jin H., Jia H., and Fang J., 2020, Brassinosteroid Regulates 3-Hydroxy-3-methylglutaryl CoA Reductase to Promote Grape Fruit Development, Journal of Agricultural and Food Chemistry, 68(43): 11987-11996

https://doi.org/10.1021/acs.jafc.0c04466

PMid:33059448

Zhu G.L., 1992, Research progress of brassinosterol phytohormones, Zhiwu Shenglixue Tongxun (Plant Physiology Communications), 28: 317-322 